\title{
A PSYCHOSOCIAL INTERVENTION FOR MENTAL HEALTH AMONG PEOPLE WITH HIV (PLWH): A LITERATURE REVIEW
}

\author{
Reineldis Gerans' ${ }^{1}$, Agung Waluyo ${ }^{2}$ \\ ${ }^{1,2}$ Faculty of Nursing, Universitas Indonesia, Depok Indonesia \\ * Correspondence: sanjes.gerans@gmail.com
}

\begin{abstract}
Mental health problems such as depression and anxiety are common for people living with Human Immunodeficiency Virus (PLWH). It may have a various negative effect on psychological well-being and the quality of life for PLWH. These adverse effects are in the form of worsening diseases, decreasing the immune system, non-compliance with ARV therapy, and even causing suicide. Various psychosocial interventions were developed to improve the mental health of PLWH. But it often creates confusion and misunderstanding because it seems to overlap each other. The effectiveness of this intervention has been investigated, but there is still little confirmatory research on various psychosocial intervention techniques, especially for PLWH. The study aimed at describing the psychosocial interventions for the mental health of PLWH. We conducted literature searches from multiple relevant sources. The four databases we cut included PROQUEST, PubMed, Plos One, and Ebsco. Three types of psychosocial interventions consisted of symptom-oriented interventions, supportive intervention, and meditation. Based on the kind of response there are several psychosocial intervention techniques such as cognitive behavior therapy (CBT), stress management, interpersonal therapy (IPT), peer support, psycho-education, meditation, relaxation, and mindfulness. There is no difference in the effectiveness of all types and intervention techniques in improving the mental health of PLWH. Psychosocial interventions proved to have a positive effect on the mental health of PLWH. Psychosocial interventions can be combined in PLHIW therapy programs with mental health problems
\end{abstract}

Keywords: PLWH, psychosocial interventions, mental health problems.

International Journal of Nursing and Health Services (IJNHS), September 2019, Volume 2, Issue 3; Page 45-57

Received: 06 April 2019; Revised: 23 April 2019; Accepted: 01 May 2019

DOI: http//doi.org/10.35654/ijnhs.v2i3.118

\section{Introduction}

Human immunodeficiency virus (HIV) was discovered in the early 1980s and has become one of the biggest health problems in the world today (1). HIV/AIDS is a significant burden on the health of the population and causes further socio-economic issues for individuals, families, communities, and governments in many countries (2). Although treatment progress is significantly influential, it remains a source of stress for many people and is associated with an increase in the level of depression. Depression is one of the most common comorbidities in HIV infection, with a prevalence of $20-37 \%$ among people living 
with HIV or 3 times higher than the general population and the incidence of anxiety disorders around 20\% (3-4).

HIV patients have been struggling with various social problems such as stigma, poverty, depression, substance abuse, and cultural beliefs. It might affect not only from the aspect of physical health but also from a mental and social health perspective that causes many problems in patient's activities and interests (5). Other difficulties including anxiety, stress, and depression could impact the quality of life of PLWH, reduce the immune system, worsening disease progression, and increased behavior at risks such as non-adherence to treatment, inconsistency in condom use, drug abuse, and even suicide (6-10).

Considering the adverse effects caused by mental health disorders among PLWH, proper treatment and treatment are needed. Many psychosocial interventions, such as cognitive-behavioral therapy (CBT), supportive intervention, meditation, and stress management, have been developed for PLWH with mental disorders. The selection and implementation of appropriate psychosocial interventions can help PLWH with mental disorders achieve optimal mental health and have an impact on improving their quality of life. The effectiveness of various psychosocial interventions is significant, which one is the most specific in enhancing the mental health of PLWH. Therefore, the most effective interventions are offered to be applied. Also, it is essential to know the psychological results of each of these interventions. However, there is often confusion and misunderstanding about the various psychosocial interventions because, in some studies, the implementation is often combined between several responses, so that overlap appears. Although the effectiveness of psychosocial interventions has investigated, there are still a few confirmatory studies on the efficacy of this intervention for PLWH.

\section{Objective}

The study aimed at describing the psychosocial interventions for the mental health of PLWH.

\section{Methods}

\subsection{Eligibility criteria}

The literature review used PRISMA to describe psychosocial interventions for the mental health of PLWH. We involved studies, including several meta-analyses, systematic reviews, and randomized controlled trial (RCT) methods.

\subsection{Search strategy}

We conducted literature searches from various sources that are relevant to the topics we discuss. Search uses the keywords "psychosocial interventions", "mental health", "PLWH", "depression", "CBT", "mindfulness", "meditation", "relaxation", "peer support", "interpersonal therapy", "stress management "," psycho-education "," meta-analysis "," systematic review ", and" Randomized Controlled trial".

\subsection{Study selection}

The four databases included PROQUEST, Plos One, PubMed, and Ebsco. We use published articles in English, full text, published in 10 years (2008-2018). After issuing similar or duplicate articles, relevant articles took for analysis. 


\section{RESULTS}

\subsection{Description of research}

The results of the study showed only 25 relevant articles reviewed from 185 including 14 meta-analyzes, 7 RCT studies, two meta-analysis studies of RCTs, and two systematic review studies and meta-analysis

\subsection{Psychosocial interventions for the mental health of PLWH}

4.2.1 Definition of psychosocial interventions

Psychosocial Interventions include interventions consisting of psychological and social elements. Psychosocial Interventions defined as interventions that involve the influence of social factors on human interaction behavior (11). This intervention consists of psycho-education, interpersonal therapy, cognitive behavioral therapy, meditation, and much more

\subsubsection{Types of psychosocial interventions}

The previous study explained that there are various psychosocial intervention techniques including (11)

a. Symptom-oriented intervention is a psychosocial intervention that focused on the symptoms experienced by the patient. Psychosocial intervention techniques included in this type; CBT, relaxation, stress management, motivational interviewing, and interpersonal therapy.

b. Supportive intervention that focused on providing motivation or support in dealing with their mental health problems. It obtained from within the patient himself or peer, health worker, and family. Psychosocial intervention techniques included in this type are peer support, Family support, and psycho-education.

c. Meditation intervention that patient's attention is diverted to a particular reference point that can provide a relaxing effect. Psychosocial intervention techniques included in this type include meditation, mindfulness, and relaxation.

\subsubsection{Psycho-social intervention techniques}

Based on the three types of psychosocial interventions, we describe the following various psychosocial intervention techniques:

a. Cognitive Behavioral Therapy (CBT)

CBT is a psychosocial intervention aimed at improving mental health. CBT focused on managing the cognitive distortions changed and increased emotional regulation (11-12) and the development of personal coping with the goal of problem solving. It was initially designed to treat depression, but its use has expanded to include the treatment of several mental health conditions, including anxiety (14-15).

CBT is a problem-focused and action-oriented therapy, which means this therapy, is used to treat specific problems associated with diagnosed mental disorders. The role of the therapist is to help clients find and practice strategies that are effective in overcoming obstacles and reducing symptoms of the disease (16). Often recommended in combination with drugs to treat conditions such as severe obsessive-compulsive disorder, opioid use disorders, bipolar disorder, and psychotic disorders (14). A study recommended the CBT and interpersonal psychotherapy for psychosocial treatments of choice (17). 
A systematic review showed that out of 62 RCT psychosocial interventions given to PLWH, CBT was the most intervention (11). This study shows CBT is one of the psychosocial interventions that are effective in improving the mental health of PLWH. It can reduce the level of depression and stress so that it ultimately enhances the quality of life for PLWH.

\section{b. Stress management}

It is a skill that allows someone to anticipate, prevent, manage, and recover from stress. This intervention study in people with HIV is usually able to improve psychological function and overcome impaired self-efficacy and reduce stress levels (18). The goals of stress management to control a person's stress level, especially chronic stress, usually to improve daily functioning. Stress produces many physical and mental symptoms that vary according to individual situational factors. It can include a decrease in physical health and an increased incidence of depression. The stress management process is one of the keys to a happy and successful life in modern society (19).

Other stress-reduction techniques include training in social activities, cognitive therapy, conflict resolution, hobbies, meditation, mindfulness, listening to music, deep breathing relaxation, yoga, reading novels, prayer, relaxation techniques. Stress management techniques will vary according to the philosophical paradigm (19). Randomized controlled studies of the effectiveness of stress management interventions in PLWH with depression and the general population of PLWH showed a decrease in anxiety and depression and an increase in quality of life (20-28).

\section{c. Interpersonal therapy}

The Interpersonal Psychotherapy (IPT) was defined as short psychotherapy to solve the intrapersonal issue. This treatment is empirically supported, which follows a very structured and time-limited approach and is intended to complete within 12-16 weeks. IPT based on the principle that there is a reciprocal relationship between life events and moods. IPT developed by Gerald Klerman and Myrna Weissman for severe depression in the 1970s and adapted for other mental disorders (29). IPT is an empirically validated intervention for depressive disorders and is more effective when used in combination with psychiatric drugs. Cognitive-behavioral therapy (CBT) and IPT are recommended in treatment guidelines as psychosocial treatments of choice, (30-31). IPT and CBT are the only psychosocial interventions in which psychiatry in the United States is mandated to be trained in professional practice (32).

The effectiveness of IPT was proved for improving the mental health of PLWH. In some RCT studies, IPT interventions increased the psychological well being of PLWH with depression and lowered their depression levels (33). Another study by Peterson et al., 2014 in populations of PLWH with depression also showed a decrease in anxiety and depression.

\section{d. Peer Support}

Peer support is an inclusive model that creates space for all people to be fully themselves who can help restructure a broader system. Peer support can offer a culture of health and abilities that are contrary to the perception of "disease" and disability (35). The main objective is to responsibly challenge assumptions about mental illness and at the same time to validate individuals for who they are and where they come. Peers support 
interventions should think creatively and not judge about the way individuals experience and make their life meanings different from making all actions and feelings diagnosed and labeled (35).

Some concepts in peer support need to be considered to achieve maximum results in its implementation. Peer support must be able to turn Oppression into awareness, Create Dialogue, Honest Direct Communication. In addition, they must understand Flexible Boundaries, Shared Strength Responsibility, and Creating New Ways.

This concept is realized through actions such as; Participatory Listening, Telling Stories, and Rebuilding Yourself. Also, Building a Joint Empowerment Relationship through Shared Responsibility and Joint Strengths, Managing Conflicts, Strengthening Peer Oversight, and Evaluation (36).

A meta-analysis study also showed the positive effect of peer support on reducing the depressive symptoms among interventions group than the control group who received the usual care (37).

\section{e. Psycho-education}

Psycho-education is an intervention given to individuals, families, and groups. This strategy focused on increasing the knowledge, helping participants to develop sources of social support, and developing coping skills to deal with those challenges. (38). Psychoeducation interventions (PE) can be applied not only to individuals but also to families and groups. Psycho-education used as part of the process of treatment and rehabilitation for patients with mental health disorders. Psycho-education was often given to patients with psychiatric disorders, including family members and people who have an interest in caring for these patients.

Walsh (2010) (38), described the psycho-education definition as follows:
a. Educate participants about challenges in life
b. Helping participants to develop social and other sources of support to face life's challenges
c. Develop coping skills to face life's challenges
d. Develop emotional support
e. Reducing the sense of stigma from participants. Changing the attitudes and beliefs of participants to a disorder,
f. Identify and explore feelings for an issue
g. Develop problem-solving skills.
h. Develop crisis-intervention skills

Psycho-education aimed not only for treatment but also for rehabilitation. It related to teaching someone about a problem so they can reduce the stress associated with the problem and prevent the problem from happening again. Psycho-education is more focused on the present and future than on past difficulties. According to Walsh (2010), psycho-education can be a single intervention, but also often combined with several other interventions to help participants face particular life challenges. Psycho-education is not the same as psychotherapy, although sometimes there is an overlap between the two interventions.

A previous study mentioned that psychotherapy was the process of interaction between a professional and his client to reduce distress, disability, malfunction of the client system in cognitive, affective, and behavioral functions (38). Randomized controlled 
studies of the effectiveness of psycho-education in 80 PLWH in the USA showed an increase in psychological well-being. Also, in the third-month evaluation, there was a decrease in anxiety and depression levels (21). It shows the importance of psycho-education as a conventional therapy for PLWH who experience mental disorders.

\section{f. Meditation}

Meditation is an individual practice that focused on attention on particular objects train mindfulness and awareness and achieves a stable and emotionally calms mental state (39-44). Meditation has practiced since ancient times in various religious traditions and beliefs, often as part of the path to enlightenment and self-realization. Meditation can be used to reduce stress, anxiety, depression, and pain, and increasing peace, perception, selfconcept, and well-being (45-46).

Two meditation methods are consisting of focus attention methods (FA), requiring the concentration of voluntary attention on selected objects, breathing, images, or words. The second is open-monitoring methods (OM) or mindfulness, which involve non-reactive monitoring of content experiences over time. (47). But in practice, these two methods are often combined. Previous studies showed a positive effect on decreasing levels of anxiety and depression and increasing the quality of life HIV-infected patients who received meditation interventions (48-49).

\section{g. Relaxation}

Relaxation in psychology is the emotional state of living beings, low tension, where no passion can come from sources such as anger, anxiety, or fear. According to the Oxford dictionary (2014) (44), relaxation is when the body and mind are free of tension and anxiety. Relaxation can be achieved through meditation, autogenic, and progressive muscle relaxation. Relaxation helps overcome stress, which is known to stress as a significant cause of mental problems and physical problems (50).

Relaxation techniques were classified into Physical, Mental, or Therapy methods. The first is physical relaxation techniques; breathing techniques are one of the easiest ways to reduce stress. It required a little effort and can be done anywhere at any time. Proper breathing techniques that combine deep breathing proved to reduce physical symptoms of depression, anxiety, and hypertension, as well as signs of emotional anger and nervousness every day (51). Progressive muscle relaxation is a relaxation technique that requires a person to focus on flexing and holding a particular set of muscles and then slowly loosening the same muscles. When individuals flex and release the muscles from top to bottom, they will feel deep relaxation (52). Progressive muscle relaxation is currently used in clinical and non-clinical settings to reduce the effects of anxiety and insomnia due to stress. The long-term goal of relaxation technique such as identify muscles problems and to relax the muscles from stress (52).

The second is mental technique; This meditation has long been practiced in other regions throughout the world. Research has shown that in addition to reducing physiological and psychological stress on your body, individuals who practice meditation have fewer doctor visits for physical and mental illness (53). Relaxation therapy for hypnosis has become another technique by health professionals to promote relaxation. When done correctly, hypnosis can put someone in a state of deep relaxation. During this 
situation, individuals are susceptible to suggestions expressed by people who do hypnosis. In addition, to relax the body, hypnosis therapy is being used to treat various conditions (53).

The third is therapeutic relaxation; this technique used in therapy by a certified counselor or therapist can include one of the previous methods discussed. Professionals in the field of psychology or counseling will have the ability to manage these various techniques. If they feel the need, they may prescribe medication to help patients get a relaxing effect. Relaxation helps a lot of disorders that can occur in a person's mental health. There are a calm mood and lower anxiety in those who practice relaxation techniques (50). Although relaxation cannot eliminate chronic diseases, it helps reduce the symptoms a person might experience. A study examining the relaxing effect of PLWHA with depression and HIV patients taking antiretroviral therapy showed a positive impact on reducing depression levels and increasing ART adherence. (54).

\section{h. Mindfulness}

Mindfulness-based stress reduction (MBSR) is a standard eight-week program that combines several components of meditation. It teaches skills to increase awareness and acceptance of experience over time, including painful emotions and physical discomfort (55).

This is the attitude of full self-awareness in the present, not looking into the past or future. Mindfulness was popularized by Jon Kabat-Zinn, a professor at the Faculty of Medicine at the University of Massachusetts, through the MBSR program he founded. Mindfulness has the same effect as meditation; don't have to sit quietly close your eyes and adjust your breathing. The practice of mindfulness includes the use of the five senses in feeling all the activities we do without judging or labeling what is being done (55).

Research has found MBSR to be an active component for managing various medical conditions, including chronic pain. Accumulated evidence shows MBSR is also useful in reducing depression and perceived stress and increasing positive effects in the general population (56). Besides that person who can cope with significant life stress from HIV (5759). One study found that HIV-positive patients with MBSR interventions had significantly lower levels compared to participants in the usual care control conditions. (58).

Some evidence suggests MBSR can increase the number of CD4 + T cells, which will be an essential immunological benefit. Another study showed participants with HIV who received MBSR had risen dramatically to $20 \mathrm{CD} 4+$ cell-T / $\mu$ l compared with a mean decrease of 185 CD $4+$ T-cells / $\mu$ in control group (60). Also, a study showed patients with HIV who had not yet received ART, MBSR seemed to have the highest potential to cure some self-reported medical symptoms. (61).

\section{Discussion}

Various Techniques Psychosocial interventions were developed to improve the mental health of everyone, and through multiple studies, it turned out that these interventions also proved to be useful for PLWH. As many as 62 randomized controlled studies in a systematic review and meta-analysis conducted by Leunen et al. (2017) (11), there are many psychosocial intervention techniques, but only divided into three types. The three types of interventions are; Symptom-oriented intervention, Supportive intervention, and Meditation intervention. Of the 62 articles, decreased levels of anxiety, 
stress, and depression, as well as an increase in psychological well-being and quality of life. Although with different levels based on the quality of the article.

Analysis of the types of psychosocial interventions carried out by Leunen et al., (2017) (11), showed no difference in effectiveness in patients with psychological disorders such as depression, or patients with medical diseases from such interventions such as CBT, interpersonal therapy, supportive therapy, and problems -solving therapy $(29,30)$. It shows that some types of psychosocial interventions are useful in improving mental health. However, it is necessary and exciting to study further the comparison of the kinds of interventions with their effects on PLWH.

Effectiveness Analysis of psychosocial interventions for mental health in the study by Leunen et al., (2017) (11), showing the focus of the intervention to determine the effectiveness of the intervention. When psychosocial interventions are given to PLWH with depressive symptoms, the benefits are felt to be higher than those with PLWH without symptoms of depression. The meta-analysis of PLHIV with high anxiety found that participants felt a more significant interest from the management of stress given (62). Likewise, in studies focusing on mental health, the effect is more significant than the study with a primary focus on non-mental health.

The intervention provider also influences the effectiveness of the intervention. Leunen et al., (2017) (11) described studies whose intervention was carried out by psychologists or psychotherapists had a significant effect than if the intervention was a counselor, co-worker, or computer. This shows that what psychosocial intervention techniques are used to treat mental health problems for PLWHA, and the critical ingredients for success are interventions. Psychologists have extensive knowledge and experience in dealing with mental health problems compared to most intervention providers who are only trained to provide intervention. However, previous meta-analyses of CBT in PLWH with depression and anxiety showed no difference in effects on groups whose response was provided by psychologists and trained research staff (63). The further research needed to prove that the psychologists or psychotherapists have higher quality interventions to solve mental health problems for PLWH.

The duration of treatment or administration of intervention also determines the effectiveness of the response. According to Leunen et al., (2017) (11) studies with a duration of treatment ranging from 12-18 hours have a more significant effect than the length of short or longer treatments. However, an RCT that compared the effectiveness of a concise CBT and standard CBT in PLWH with depression and anxiety showed similar effectiveness (64). Several meta-analyses that investigated the efficacy of psychological interventions for depression and anxiety for PLWH also found no difference between short or long duration treatments $(29-30,63)$. On the other hand, an online CBT RCT in patients with chronic somatic and depression does indeed find the effect of the highest duration of treatment at a more extended period (65). Therefore it is not clear whether the length of the intervention affects the effectiveness of the response. On mental health problems, the severity of symptoms needs to be considered. Maybe PLWH with symptoms of a more severe mental disorder needs a longer duration than those with PLWH with mild or moderate symptoms. For this matter, further research is required.

The application of psychosocial interventions in various studies often confuses because it seems to overlap with each other. For example, in stress management interventions, there are different intervention techniques, including meditation and 
relaxation, whereas relaxation intervention is the result of meditation and mindfulness techniques. Similarly, the implementation of mindfulness interventions also involves meditation techniques. Therefore, the application often requires several interventions at the same time to get the optimal result (11). Even so, it is essential to remember that not all techniques will have the same effect for everyone. That is why applying various psychosocial intervention techniques is very important to find what methods are most suitable for patients. Also, further research needed to confirm the effectiveness of all existing psychosocial interventions on improving the mental health of PLWH in the future.

\section{Conclusions}

We concluded that there are many types and psychosocial intervention techniques that can improve the mental health of PLWH. There were no differences in the effects of the three types of psychosocial interventions, both symptom-based interventions, supportive interventions, and meditation. The three types of interventions have a positive impact on the mental health of PLWH, even at different levels. Thus psychosocial interventions are critical as an intervention choice and are combined in the care and treatment of PLWH with mental health problems.

\section{References}

1. World Health Organization. Policy brief: Consolidated guidelines on HIV prevention, diagnosis, treatment, and care for key populations. World Health Organization; 2017.

2. Lari MA, Faramarzi H, Shams M, Marzban M, Joulaei H. Sexual dysfunction, depression, and quality of life in patients with HIV infection. Iranian journal of psychiatry and behavioral sciences. 2013;7(1):61.

3. Donnerer J, Haas BJ, Kessler HH. Single-measurement therapeutic drug monitoring of the HIV/AIDS drugs abacavir, zidovudine, lamivudine, efavirenz, nevirapine, lopinavir, and nelfinavir. Pharmacology. 2008;82(4):287-92.

4. Chen WT, Shiu CS, Yang JP, Lee SY, Lee TS, Simoni JM, Bao MJ, Lu HZ. Fatigue and sleep disturbance related to perceived stress in Chinese HIV-positive individuals: A mixed-methods study. Journal of AIDS \& clinical research. 2013 Jun 18;4(6).

5. Bhargava S, Sawant K. Drought stress adaptation: metabolic adjustment and regulation of gene expression. Plant Breeding. 2013 Feb;132(1):21-32.

6. Nokes K, Johnson MO, Webel A, Rose CD, Phillips JC, Sullivan K, Tyer-Viola L, Rivero-Méndez M, Nicholas P, Kemppainen J, Sefcik E. Focus on increasing treatment self-efficacy to improve human immunodeficiency virus treatment adherence. Journal of Nursing Scholarship. 2012 Dec;44(4):403-10.

7. Walkup JT, Albano AM, Piacentini J, Birmaher B, Compton SN, Sherrill JT, Ginsburg GS, Rynn MA, McCracken J, Waslick B, Iyengar S. Cognitive behavioral therapy, sertraline, or a combination in childhood anxiety. New England Journal of Medicine. 2008 Dec 25;359(26):2753-66.

8. Owe-Larsson MB, Säll L, Salamon E, Allgulander C. HIV infection, and psychiatric illness. African journal of psychiatry. 2009;12(2).

9. Brion JM, Leary MR, Drabkin AS. Self-compassion and reactions to serious illness: The case of HIV. Journal of health psychology. 2014 Feb;19(2):218-29.

10. Crane J. Adverse events and placebo effects: African scientists, HIV, and ethics in the 'global health sciences.' Social Studies of Science. 2010 Dec;40(6):843-70. 
11. Van Luenen S, Garnefski N, Spinhoven P, Spaan P, Dusseldorp E, Kraaij V. The benefits of psychosocial interventions for mental health in people living with HIV: a systematic review and meta-analysis. AIDS and Behavior. 2018 Jan 1;22(1):9-42.

12. Beck JS. Cognitive behavior therapy: Basics and beyond. Guilford Press; 2011 Aug 18.

13. Kircher T, Arolt V, Jansen A, Pyka M, Reinhardt I, Kellermann T, Konrad C, Lueken U, Gloster AT, Gerlach AL, Ströhle A. Effect of cognitive-behavioral therapy on neural correlates of fear conditioning in panic disorder. Biological Psychiatry. 2013 Jan 1;73(1):93-101.

14. Bogart LM, Wagner GJ, Mutchler MG, Risley B, McDavitt BW, McKay T, Klein DJ. Community HIV treatment advocacy programs may support treatment adherence. AIDS Education and Prevention. 2012 Feb;24(1):1-4.

15. Zhipei ZH, Zhang L, Jiang J, Wei LI, Xinyi CA, Zhirui ZH, Zhang T, Chunbo LI. Comparison of psychological placebo and waiting list control conditions in the assessment of cognitivebehavioral therapy for the treatment of generalized anxiety disorder: a meta-analysis. Shanghai archives of psychiatry. 2014 Dec;26(6):319.

16. Schacter DL, Gilbert DT, Wegner DM (2010), Psychology (2nd ed.), New York: Worth Pub, p. 600)

17. Barth J, Munder T, Gerger H, Nüesch E, Trelle S, Znoj H, Jüni P, Cuijpers P. Comparative efficacy of seven psychotherapeutic interventions for patients with depression: a network metaanalysis. Focus. 2016 Apr 7;14(2):229-43.

18. Brown JL, Vanable PA. Cognitive-behavioral stress management interventions for persons living with HIV: a review and critique of the literature. Annals of Behavioral Medicine. 2008 Feb 1;35(1):26-40.

19. Susic P. Stress Management: What can you do?. St. Louis Psychologists and Counseling Information and Referral. 2013.

20. Berger BE, Ferrans CE, Lashley FR. Measuring stigma in people with HIV: Psychometric assessment of the HIV stigma scaleף. Research in nursing \& health. 2001 Dec;24(6):518-29.

21. Fife BL, Scott LL, Fineberg NS, Zwickl BE. Promoting adaptive coping by persons with HIV disease: evaluation of a patient/partner intervention model. Journal of the Association of Nurses in AIDS Care. 2008 Jan 1;19(1):75-84.

22. Heckman TG, Heckman BD, Kochman A, Sikkema KJ, Suhr J, Goodkin K. Psychological symptoms among persons 50 years of age and older living with HIV disease. Aging \& mental health. 2002 May 1;6(2):121-8.

23. Lengacher CA, Johnson-Mallard V, Post-White J, Moscoso MS, Jacobsen PB, Klein TW, Widen RH, Fitzgerald SG, Shelton MM, Barta M, Goodman M. Randomized controlled trial of mindfulness-based stress reduction (MBSR) for survivors of breast cancer. Psycho-Oncology: Journal of the Psychological, Social, and Behavioral Dimensions of Cancer. 2009 Dec;18(12):1261-72.

24. Streeter CC, Whitfield TH, Owen L, Rein T, Karri SK, Yakhkind A, Perlmutter R, Prescot A, Renshaw PF, Ciraulo DA, Jensen JE. Effects of yoga versus walking on mood, anxiety, and brain GABA levels: a randomized controlled MRS study. The Journal of Alternative and Complementary Medicine. 2010 Nov 1;16(11):1145-52.

25. Klein J, Cook-Cottone C. The effects of yoga on eating disorder symptoms and correlates: A review. International Journal of Yoga Therapy. 2013 Jan 1;23(2):41-50.

26. Liu L, Pang R, Sun W, Wu M, Qu P, Lu C, Wang L. Functional social support, psychological capital, and depressive and anxiety symptoms among people living with HIV/AIDS employed full-time. BMC psychiatry. 2013 Dec;13(1):324.

27. Relf MV, Huang B, Campbell J, Catania J. Gay identity, interpersonal violence, and HIV risk behaviors: An empirical test of theoretical relationships among a probability-based sample of 
urban men who have sex with men. Journal of the Association of Nurses in AIDS Care. 2004 Mar 1;15(2):14-26.

28. Vranceanu AM, Safren SA, Lu M, Coady WM, Skolnik PR, Rogers WH, Wilson IB. The relationship of post-traumatic stress disorder and depression to antiretroviral medication adherence in persons with HIV. AIDS patient care and STDs. 2008 Apr 1;22(4):313-21.

29. Cuijpers P, Cristea IA, Karyotaki E, Reijnders M, Huibers MJ. How effective are cognitive behavior therapies for major depression and anxiety disorders? A meta-analytic update of the evidence. World Psychiatry. 2016 Oct;15(3):245-58.

30. Cuijpers P, Berking M, Andersson G, Quigley L, Kleiboer A, Dobson KS. A meta-analysis of cognitive-behavioral therapy for adult depression, alone and in comparison with other treatments. The Canadian Journal of Psychiatry. 2013 Jul;58(7):376-85.

31. Andersson G, Cuijpers P. Internet-based, and other computerized psychological treatments for adult depression: a meta-analysis. Cognitive behavior therapy. 2009 Dec 1;38(4):196-205.

32. Hollon, Steven D.; Beck, Aaron T. (2013). Cognitive and Cognitive-Behavioral Therapies. In Lambert, Michael J. Bergin and Garfield's Handbook of Psychotherapy and Behavior Change (6th ed.). Hoboken, NJ: John Wiley \& Sons. pp. 393-442. ISBN 978-1-118-41868-0.

33. Ransom D, Heckman TG, Anderson T, Garske J, Holroyd K, Basta T. Telephone-delivered, interpersonal psychotherapy for HIV-infected rural persons with depression: a pilot trial. Psychiatric Services. 2008 Aug;59(8):871-7

34. Gold JA, Grill M, Peterson J, Pilcher C, Lee E, Hecht FM, Fuchs D, Yiannoutsos CT, Price RW, Robertson K, Spudich S. Longitudinal characterization of depression and mood states beginning in primary HIV infection. AIDS and Behavior. 2014 Jun 1;18(6):1124-32.

35. Edwards A, Curtis S, Sherrard J. Survey of risk behavior and HIV prevalence in an English prison. International Journal of STD \& AIDS. 1999 Jul 1;10(7):464-6.

36. Mead GE, Morley W, Campbell P, Greig CA, McMurdo M, Lawlor DA. Exercise for depression. Cochrane database of systematic reviews. 2008(4).

37. Pfeiffer PN, Heisler M, Piette JD, Rogers MA, Valenstein M. Efficacy of peer support interventions for depression: a meta-analysis. General hospital psychiatry. 2011 Jan 1;33(1):29-36.

38. Walsh, Joseph. 2010. Psychoeducation In Mental Health. Chicago: Lyceum Books, Inc.

39. Walsh R, Shapiro SL. The meeting of meditative disciplines and Western psychology: a mutually enriching dialogue. American psychologist. 2006 Apr;61(3):227.

40. Cahn BR, Polich J. Meditation states and traits: EEG, ERP, and neuroimaging studies. Psychological bulletin. 2006 Mar;132(2):180.

41. Jevning R, Wallace RK, Beidebach M. The physiology of meditation: a review. A wakeful hypometabolic integrated response. Neuroscience \& Biobehavioral Reviews. 1992 Sep 1;16(3):415-24.

42. Goleman, Daniel (1988). The meditative mind: The varieties of meditative experience. New York: Tarcher. ISBN 978-0-87477-833-5

43. Webster M. Merriam-Webster's collegiate dictionary. Springfield, MA: Merriam-Webster; 2003 Jul.

44. Baldick C. The Oxford dictionary of literary terms. OUP Oxford; 2015 May 14.

45. Goyal M, Singh S, Sibinga EM, Gould NF, Rowland-Seymour A, Sharma R, Berger Z, Sleicher D, Maron DD, Shihab HM, Ranasinghe PD. Meditation programs for psychological stress and well-being: a systematic review and meta-analysis. JAMA internal medicine. 2014 Mar 1;174(3):357-68. 
46. Shaner L, Kelly L, Rockwell D, Curtis D. Calm abiding: the lived experience of the practice of long-term meditation. Journal of Humanistic Psychology. 2017 Jan;57(1):98-121.

47. Lutz A, Slagter HA, Dunne JD, Davidson RJ. Attention regulation and monitoring in meditation. Trends in cognitive sciences. 2008 Apr 1;12(4):163-9.

48. Busman RA, Page C, Oka E, Giordani B, Boivin MJ. Factors contributing to the psychosocial adjustment of Ugandan preschool children with HIV/AIDS. Neuropsychology of children in Africa 2013 (pp. 95-115). Springer, New York, NY.

49. Chhatre S, Metzger DS, Frank I, Boyer J, Thompson E, Nidich S, Montaner LJ, Jayadevappa R. Effects of behavioral stress reduction Transcendental Meditation intervention in persons with HIV. AIDS Care. 2013 Oct 1;25(10):1291-7.

50. Pagnini F, Manzoni GM, Castelnuovo G, Molinari E. A brief literature review about relaxation therapy and anxiety. Body, Movement, and Dance in Psychotherapy. 2013 May 1;8(2):71-81.

51. MacGregor, H.E. (2004). Take a breath to relieve stress specialized breathing techniques beneficial to health, practitioners say. Journal-Gazette. Retrieved from http://search.proquest.com/docview/411159521

52. Greenwald, D. (2012, May 24). Innovative Health: De-stress with a progressive muscle relaxation technique. Alamogordo Daily News. Retrieved from http://search.proquest.com/docview/1015669126

53. Faílde Garrido JM, Lameiras Fernández M, Foltz M, Rodríguez Castro Y, Carrera Fernández MV. Cognitive performance in men and women infected with HIV-1. Psychiatry journal. 2012 Dec 26;2013.

54. Chen WT, Shiu CS, Yang JP, Lee SY, Lee TS, Simoni JM, Bao MJ, Lu HZ. Fatigue and sleep disturbance related to perceived stress in Chinese HIV-positive individuals: A mixed-methods study. Journal of AIDS \& clinical research. 2013 Jun 18;4(6).

55. Robinson FP, Mathews HL, Witek-Janusek L. Psycho-endocrine-immune response to mindfulness-based stress reduction in individuals infected with the human immunodeficiency virus: a quasi-experimental study. The Journal of Alternative \& Complementary Medicine. 2003 Oct 1;9(5):683-94.

56. Nyklíček I, Kuijpers KF. Effects of a mindfulness-based stress reduction intervention on psychological well-being and quality of life: are increased mindfulness indeed the mechanism?. Annals of Behavioral Medicine. 2008 Jun 1;35(3):331-40.

57. Ledesma D, Kumano H. Mindfulness-based stress reduction and cancer: a meta-analysis. Psycho-Oncology: Journal of the Psychological, Social, and Behavioral Dimensions of Cancer. 2009 Jun;18(6):571-9.

58. Gayner B, Esplen MJ, DeRoche P, Wong J, Bishop S, Kavanagh L, Butler K. A randomized controlled trial of mindfulness-based stress reduction to manage affective symptoms and improve quality of life in gay men living with HIV. Journal of behavioral medicine. 2012 Jun 1;35(3):272-85.

59. Duncan LG, Moskowitz JT, Neilands TB, Dilworth SE, Hecht FM, Johnson MO. Mindfulnessbased stress reduction for HIV treatment side effects: a randomized, wait-list controlled trial. Journal of Pain and symptom management. 2012 Feb 1;43(2):161-71.

60. Creswell JD, Myers HF, Cole SW, Irwin MR. Mindfulness meditation training effects on CD4+ T lymphocytes in HIV-1 infected adults: A small randomized controlled trial. Brain, behavior, and immunity. 2009 Feb 1;23(2):184-8.

61. SeyedAlinaghi S, Jam S, Foroughi M, Imani A, Mohraz M, Djavid GE, Black DS. A randomized controlled trial of mindfulness-based stress reduction delivered to human immunodeficiency virus-positive patients in Iran: effects on $\mathrm{CD}^{+} \mathrm{T}$ lymphocyte count and medical and psychological symptoms. Psychosomatic medicine. 2012;74(6):620-7. 
62. Scott-Sheldon LA, Kalichman SC, Carey MP, Fielder RL. Stress management interventions for HIV+ adults: a meta-analysis of randomized controlled trials, 1989 to 2006. Health Psychology. 2008 Mar;27(2):129.

63. Herbst JH, Jacobs ED, Finlayson TJ, McKleroy VS, Neumann MS, Crepaz N, HIV/AIDS Prevention Research Synthesis Team. Estimating HIV prevalence and risk behaviors of transgender persons in the United States: a systematic review. AIDS and Behavior. 2008 Jan 1;12(1):1-7.

64. Meuldijk D, Carlier IV, Van Vliet IM, Van Veen T, Wolterbeek R, van Hemert AM, Zitman FG. The clinical effectiveness of concise cognitive behavioral therapy with or without pharmacotherapy for depressive and anxiety disorders; a pragmatic randomized controlled equivalence trial in clinical practice. Contemporary clinical trials. 2016 Mar 1;47:131-8.

65. van Straten A, Geraedts A, Verdonck-de Leeuw I, Andersson G, Cuijpers P. Psychological treatment of depressive symptoms in patients with medical disorders: a meta-analysis. Journal of Psychosomatic Research. 2010 Jul 1;69(1):23-32. 